\title{
Correction to: A Generalized Fejér's Theorem for Locally Compact Groups
}

\section{Huichi Huang ${ }^{1}$}

Published online: 29 January 2019

(c) Mathematica Josephina, Inc. 2019

\section{Correction to: J Geom Anal (2018) 28:909-920 https://doi.org/10.1007/s12220-017-9847-7}

As pointed out by Hanfeng Li, we fix a gap in Theorem 1.2.

There are three issues in the paper.

(1) Let $G$ be a locally compact group with the unit $e_{G}$ and a fixed left Haar measure $\mu$. Let $v$ be the right Haar measure given by $v(A)=\mu\left(A^{-1}\right)$ for every Borel subset $A$ of $G$.

Theorem 1.2 is stated as follows.

Theorem (A generalized Fejér's theorem)

Consider a locally compact group $G$ with a fixed left Haar measure $\mu$ and the corresponding right Haar measure v. Let $\left\{F_{\theta}\right\}_{\theta \in \Theta}$ be an approximate identity of $L^{1}(G)$. Assume that there exists a local partition $\left\{A_{1}, A_{2}, \ldots, A_{k}\right\}$ of $G$ such that $\lim _{\theta} \int_{A_{j}} F_{\theta}(y) d \mu(y)=\lambda_{j}$ for every $1 \leq j \leq k$.

For an $f$ in $L^{\infty}(G)$, if there exists $x$ in $G$ such that $\lim _{\substack{y \rightarrow e_{G} \\ y \in A_{j}}} f\left(y^{-1} x\right)$ (denoted by $\left.f\left(x, A_{j}\right)\right)$ exists for every $1 \leq j \leq k$, then

$$
\lim _{\theta} F_{\theta} * f(x)=\sum_{j=1}^{k} \lambda_{j} f\left(x, A_{j}\right) .
$$

The original article can be found online at https://doi.org/10.1007/s12220-017-9847-7.

$凶 \quad$ Huichi Huang

huanghuichi@cqu.edu.cn

1 College of Mathematics and Statistics, Chongqing University, Chongqing 401331,

People's Republic of China 
Moreover if $\lim _{\theta} \sup _{y \in \mathcal{N}^{c}}\left|F_{\theta}(y)\right|=0$ for any neighborhood $\mathcal{N}$ of $e_{G}$, then for every $f$ in $L^{1}(G, v)\left(\right.$ or $\left.L^{\infty}(G)\right)$ such that each $f\left(x, A_{j}\right)$ exists for some $x$ in $G$, we have

$$
\lim _{\theta} F_{\theta} * f(x)=\sum_{j=1}^{k} \lambda_{j} f\left(x, A_{j}\right)
$$

In the proof of Theorem 1.2, there are two corrections:

(a) The sentence "Now assume that $\lim _{\theta} \sup _{y \in \mathcal{N}^{c}}\left|F_{\theta}(y)\right|=0$ for any neighborhood $\mathcal{N}$ of $e_{G}$ and $f$ is in $L^{1}(G)$ such that each $f\left(x, A_{j}\right)$ exists for some $x$ in $G$ " in the line -5 on page 5 is changed to "Now assume that $\lim _{\theta} \sup _{y \in \mathcal{N}^{c}}\left|F_{\theta}(y)\right|=0$ for any neighborhood $\mathcal{N}$ of $e_{G}$ and $f$ is in $L^{1}(G, v)$ such that each $f\left(x, A_{j}\right)$ exists for some $x$ in $G$ ".

(b) the last identities are changed to:

$$
\underset{\theta}{\lim \sup }\left|\int_{\mathcal{N}^{c}} F_{\theta}(y) f\left(y^{-1} x\right) d \mu(y)\right| \leq\left[\lim _{\theta} \sup _{y \in \mathcal{N}^{c}}\left|F_{\theta}(y)\right|\right]\|f\|_{L^{1}(G, v)}=0 .
$$

(2) Statement of Corollary 2.2 is changed to the following:

Corollary Given any approximate identity $\left\{F_{\theta}\right\}_{\theta \in \Theta}$ of $L^{1}(G)$ and local partition $\left\{A_{1}, \ldots, A_{k}\right\}$ of $G$, if for an $f$ in $L^{\infty}(G)$, every $f\left(x, A_{j}\right)$ exists, then there exists a subnet $\Theta_{1}$ of $\Theta$ such that every $\lim _{\theta \in \Theta_{1}} \int_{A_{j}} F_{\theta}(y) d \mu(y)$ exists (denoted by $\lambda_{j}\left(\Theta_{1}\right)$ ) and

$$
\lim _{\theta \in \Theta_{1}} F_{\theta} * f(x)=\sum_{j=1}^{k} \lambda_{j}\left(\Theta_{1}\right) f\left(x, A_{j}\right)
$$

Moreover if $\lim _{\theta \in \Theta_{1}} \sup _{y \in \mathcal{N}^{c}}\left|F_{\theta}(y)\right|=0$ for every neighborhood $\mathcal{N}$ of $e_{G}$, then for every $f$ in $L^{1}(G, v)\left(\right.$ or $\left.L^{\infty}(G)\right)$ such that every $f\left(x, A_{j}\right)$ exists for some $x$ in $G$, we have

$$
\lim _{\theta \in \Theta_{1}} F_{\theta} * f(x)=\sum_{j=1}^{k} \lambda_{j}\left(\Theta_{1}\right) f\left(x, A_{j}\right) .
$$

(3) Before Corollary 3.5, add a definition.

For $k=\left(k_{1}, \ldots, k_{d}\right)$ in $\{0,1\}^{d}$, define

$$
J_{k}=\prod_{l=1}^{d} J_{k_{l}}
$$


with $J_{0}=(-\infty, 0)$ and $J_{1}=[0, \infty)$.

Publisher's Note Springer Nature remains neutral with regard to jurisdictional claims in published maps and institutional affiliations. 